\title{
Simulated Performance Analysis of an Optical Frequency Comb in a WDM System
}

\author{
Rifat Nazneen*, Ebad Zahir
}

\begin{abstract}
This paper presents the research work for a proposed novel scheme for generating a flat and equally spaced optical frequency comb using a simulated layout using Optisystem. Three pump sources at $1 \mathrm{~nm}$ wavelength apart are injected along with a $25 \mathrm{GHz}$ RF signal having different amplitudes into a cascade of Dual-Drive Mach-Zehnder Modulator and Electro-Absorption Modulator and multiple highly nonlinear fiber loops. The initial scheme is using Four Wave - Mixing and yields a comb of 27 lines with uneven gain and a $6 \mathrm{dBm}$ power deviation. After characterizing the response by exploring critical parameters such as pump power, frequency spacing, dispersion effect, Erbium Doped Fiber Amplifier and DC bias of the modulators, the researchers were able to reduce the power deviation down to $2 \mathrm{dBm}$ at the expense of some comb lines. This final optimized scheme was then evaluated for a preliminary Wavelength Division Multiplexing setup having only five channels/modes at $10 \mathrm{~Gb} / \mathrm{s}$ for a channel length of $1 \mathrm{~km}$. Each of the received de-multiplexed channels was evaluated through recorded data in the form of Bit Error Rate and Qualityfactor and the final results are shown to be quite promising.
\end{abstract}

Keywords - Optical Frequency Comb; Four Wave Mixing; Highly Nonlinear Fiber; Dual-Drive Mach-Zehnder Modulator; Electro-Absorption Modulator; Fiber-optic Communication; Wavelength Division Multiplexing;

\section{INTRODUCTION}

Now a day's large demand for the data rates, higher bandwidth, and high gain are required without cross talks, interface among the subscribers for communication networks. Therefore several types of research are ongoing to meet this margin. There are several conventional approaches to expanding spectral efficiency such as the implementation of individual laser sources, but it entails additional concerns regarding precise wavelength control for each channel, leading to increased management and inventory. To avoid these phenomena, Optical Frequency Comb (OFC) is one of the recent researches and demandable approach to enhance spectral efficiency, bandwidth, and can mitigate the unwanted disturbance in the communication systems.

An optical frequency comb is a pulsed laser source

\section{Rifat Nazneen}

Dept. of Electrical and Electronics Engineering

American International University- Bangladesh (AIUB)

Dhaka, Bangladesh

e-mail: syeda.rifat18@gmail.com emitting a periodic waveform with a stabilized repetition rate [1]. In the frequency domain, such a source is constituted of a discrete set of frequencies spaced by a constant period with flattening gain. It attracts the interest of researchers due to its wide applications such as precise photonic metrology, photonic arbitrary waveform generation, and ultrafast photonic signal processing [2], multi-wavelength ultra-short pulse generation [3], in optical communication systems like Wavelength Division Multiplexing (WDM), Dense Wavelength Division Multiplexing (DWDM) [4] etc. There are many purposes to generate an evenly spaced large number of frequency comb such as gain switching, mode-locked laser, nonlinearity effect in optical fibers [5], re-circulating shifter loop [6] etc. but it is difficult to flatten the spectrum. Recent research has shown that by using different modulators flat spectral lines can be produced in lower power deviations.

This paper proposes an approach which was successful in generating an optical frequency comb in two steps. Initially, the scheme generated evenly spaced 27 comb lines with fluctuations of $6 \mathrm{dBm}$ power deviation [7]. This scheme was then characterized and optimized by the effective parameters such as pump sources, channel spacing, dispersion of Single Mode Fiber (SMF) and Erbium Doped Fiber Amplifier (EDFA) lengths. Due to the presence of uneven gain in the results, a modified scheme was again proposed which yielded 15 flat evenly spaced comb lines using $25 \mathrm{GHz}$ RF signals of two different amplitudes in a cascade of Dual-Drive MachZehnder (DD-MZM) and Electro-Absorption Modulator (EAM) in a nonlinear fiber loop [8]. This scheme shows promising results of only $2 \mathrm{dBm}$ power deviation. Another advantage was that since the DD-MZM can modify both the amplitude and the phase of the modulated signal, there was no need for a phase modulator in the scheme. The scheme was evaluated for a number of comb lines and flatness of power deviation by varying the number of sources with different channel spacing. Performance evaluation was carried out by testing the Bit Error Rate (BER) and Quality Factor (Q-factor) for each of the five modes after introducing a $10 \mathrm{~Gb} / \mathrm{s}$ signal for $1 \mathrm{~km}$ transmission length which justifies the use of the modes as individual channels for a Wavelength Division Multiplexing (WDM) system. Finally, the system was optimized for the maximum transmission length.

\section{THEORY AND METHODOLOGIES}

The proposed scheme is based on the impact of both the modulation of the signals and nonlinear effect of the fibers.

\section{Ebad Zahir}

Senior Assistant Professor, Dept. of Electrical and Electronics Engineering American International University- Bangladesh (AIUB)

Dhaka, Bangladesh

e-mail: ebad.zahir@aiub.edu 
The theory behind the generation of flat evenly spaced OFC can be described by the conditions of these following effects.

\section{A. FWM}

Four-Wave Mixing (FWM) is a third order parametric nonlinear effect in the optical medium. It occurs when two or more light waves travel through the fiber and generate waves at new wavelengths [9]. If three waves at different wavelengths pass through a longer length of fiber then it will generate the fourth wavelength. Then this effect can be defined by the equation,

$$
f_{\text {new }}=f_{1}+f_{2}+f_{3}
$$

Where, $f_{1} \& f_{2}$ are pumped waves, $f_{3}=$ weak signal or probe light, and $f_{\text {new }}$ is the new photon at new wavelength or idler wavelength. This relationship is shown in Fig.1.

Pumping light

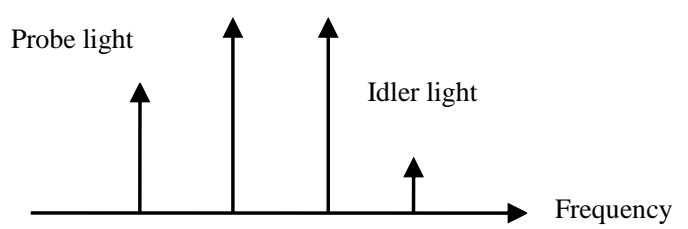

Fig. 1. Four Wave Mixing in the frequency domain

Using this FWM, the wavelength conversion occurs to provide high-efficiency conversion and broad bandwidth in the fiber which is useful for the optical comb generation. Before converting the wavelength for comb generation using FWM some conditions must be satisfied:

- Pump wavelength must coincide with zero dispersion wavelengths.

- Chromatic dispersion variation in the longitudinal direction of the fiber should be minimized

- States of polarization of the pump and signals must be matched [9].

For an efficient generation of FWM, the phase of this nonlinear polarization must match with the phase of the field wave at a new wavelength as it propagates through the fiber. This phase-matching requirement occurs when the net wavevector mismatch $\kappa=0$, where $\kappa$ is given by [10]:

$$
\kappa=\Delta \kappa_{M}+\Delta \kappa_{W}+\Delta \kappa_{N L}
$$

Where, $\Delta \kappa_{M}, \Delta \kappa_{W}$ and $\Delta \kappa_{N L}$ represent the mismatch occurring as a result of material dispersion, waveguide dispersion and nonlinear effects, respectively. Here,

$$
\kappa_{N L}=\gamma\left(P_{1}+P_{2}\right)
$$

Where, $\gamma$ is the fiber nonlinear coefficient, $P_{1}$ and $P_{2}$ are the incident power of both Lasers.

In Highly Nonlinear Fiber (HNLF) the FWM generate efficiently to provide more optical spectral lines in broad bandwidth range. By decreasing effective area $A_{\text {eff }}$ of nonlinear fiber enhance nonlinear coefficient which satisfies the phase matching conditions of the spectral lines. Therefore, multiple FWM generates in fiber to enhance the OFC.

$$
\gamma=\frac{2 \pi n_{2}}{\lambda A_{e f f}}
$$

In the loop of nonlinear fiber more sidebands are induced for the enhancement of FWM. The generation of number of sidebands depends on the equation,

$$
N=3 \times n-2
$$

Where, $n$ represents the number of pump lights launched into the feedback system and $N$ means the total number of optical waves inside the fiber.

The nonlinear effects depend on transmission length. The longer the length of fiber link, the more the light interaction, and thus, the greater the nonlinear effect which is important for the optical comb generation.

$$
L_{e f f}=\frac{1}{\alpha}
$$

\section{B. DD-MZM}

A DD-MZM is a two-arm modulator where an electric field is applied to control the phase and if the contribution in each arm is properly controlled then it becomes an intensity modulator. It requires two electrical signals, each driving the modulator at half the voltage of a single-drive device [11]. Normal operation consists of applying half of the required electrical amplitude on each RF port, RF + and RF-, with a $\pi$ phase shift between them;

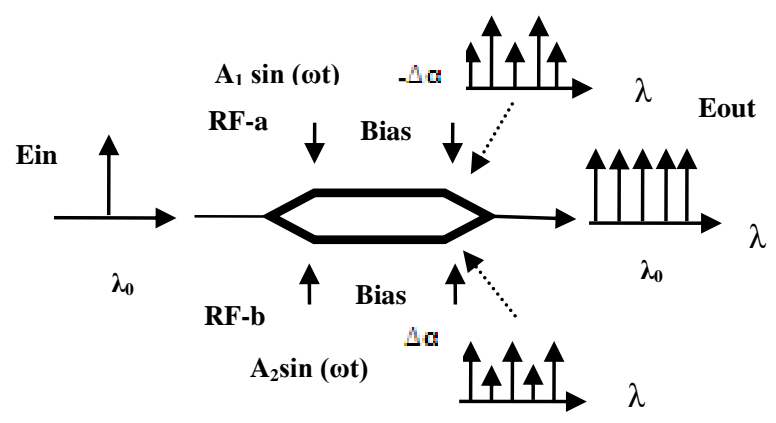

Fig. 2. General Scheme of optical frequency comb generation using a MZM

In Fig. 2, the two electrical RF signals, RF-a and RF-b, are applied on the two arms of the MZM with two different amplitudes of A1 $\sin (\omega \mathrm{t}), \mathrm{A} 2 \sin (\omega \mathrm{t})$ along with a phase shift. These two arms are biased with $-\Delta \alpha$ and $+\Delta \alpha$ so that the amplitude can be controlled. Eout can be expressed as;

$$
E_{\text {out }}(t)=\frac{E_{\text {in }}(t)}{2} \sum_{k=-\infty}^{+\infty}\left(J_{k}\left(A_{1}\right) \ell^{J\left(k w t+\alpha_{1}\right)}+J_{k}\left(A_{2}\right) \ell^{J\left(k w t+\alpha_{2}\right)}\right.
$$

By simplifying equation (7), it can define $2 \Delta \mathrm{A}$ as the peakto-peak phase difference in each arm;

$$
\Delta A=\frac{A_{1}-A_{2}}{2}
$$

Bias difference between the arms $2 \Delta \alpha$ can be defined from equation (9) 


$$
\Delta \alpha=\frac{\alpha_{1}-\alpha_{2}}{2}
$$

Combining equations (8) \& (9) can be used to generate a flat OFC;

$$
\Delta A+\Delta \alpha=\frac{\pi}{2}
$$

Also the MZM is based on electro-optic effect, which means that the refractive index, $n$, of the material changes with respect to the voltage, V, applied across the arms as well as the phase change in the signals [12]. This can be defined by equation (11):

$$
\Delta \alpha=\frac{2 \pi}{\lambda} \Delta n L
$$

\section{C. $\boldsymbol{E A M}$}

EAM is another single drive external modulator which drives the RF signal with a specific bias voltage on the plane of the waveguide [12]. The RF signal is injected on the EAM to absorb the unwanted signals and modulate the desired signal according to the operating wavelength. The general equation for EAM to modulate the output signal depends on bias voltage $\mathrm{E}(\mathrm{v})$ and can be defined by equation (12):

$$
E(v)=I(v)^{\left(1+j \alpha_{p}(v) / 2\right)}
$$

\section{Proposed Methodology of Initial Scheme}

The initial scheme was successful in generating an OFC due to the presence of a highly nonlinear feedback structure as shown in Fig 3. The scheme is divided into two parts. In the first part, three continuous wave $(\mathrm{CW})$ lasers with a linewidth of $10 \mathrm{MHz}$ are coupled together using two $3 \mathrm{~dB}$ couplers to accumulate the pump power. It then passes through the phase modulator which modulates a $20 \mathrm{GHz}$ bandwidth RF signal to suppress Stimulated Brillouin Scattering. The phase modulation of the $\mathrm{CW}$ laser source leads to the generation of many spectral sidebands by increasing the phase modulation depth and a wide range of OFC is obtained [13].

The modulated signal passes through a SMF of $1 \mathrm{~km}$ to reduce the chirp induced by the phase modulator. A short pulse train with a narrow linewidth is achieved after the SMF. This train is then amplified by an EDFA with amplified spontaneous emission (ASE) to extend the transmission distance. Finally, an optical filter is used to eliminate the ASE noise from the EDFA in order to get the desired signal.

In the second part, the focus is on the effect of multiple HNLFs on FWM generation. The feedback system contains three HNLFs which increase both multiple FWM and Self Phase Modulation (SPM) effects in the fibers to generate a broad range of OFC. In the feedback structure, a polarization combiner was used [14]. By using these two couplers, both are feeding back to the polarization combiner at $9 \%$ coupling ratio. Recent experimental work validates the use of highly nonlinear feedback loop.

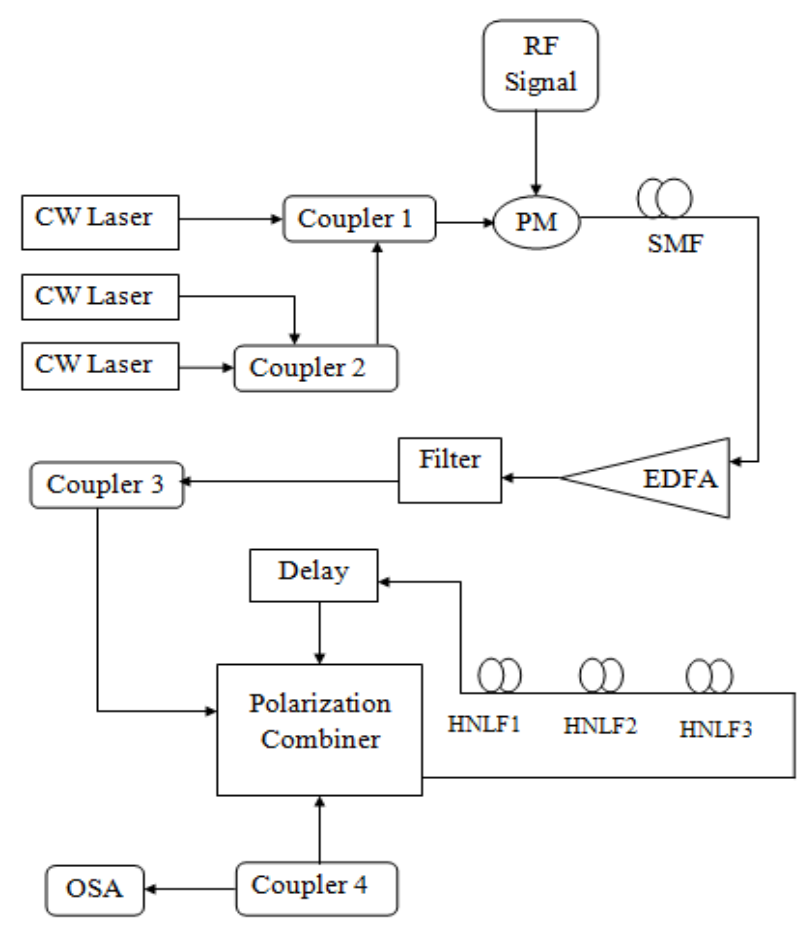

Fig. 3. Proposed Scheme for the optical frequency comb generation

Experimentally, it has been shown that with 9\% feedback ratio and two laser sources can be used to generate frequency comb lines [15]. However, when the three pumps with same frequency spacing are injected into the feedback structure, second order equally spaced sidebands are produced by FWM in highly nonlinear fibers with a specific delay between each other. This delay reduces the overlapping of the spectral lines created with the FWM in the fiber loop.

\section{RESULTS, AND DISCUSSIONS}

In the Optisystem simulated scheme, three pumps at 1553 $\mathrm{nm}, 1552 \mathrm{~nm}$, and $1551 \mathrm{~nm}$ are injected into a $3 \mathrm{~dB}$ coupler with $10 \mathrm{MHz}$ linewidth. An RF signal with $20 \mathrm{GHz}$ bandwidth is modulated with the pulse waves to change the phase. A short pulse train is generated in Optisystem with 3 ps linewidth after the SMF having $1 \mathrm{ps} / \mathrm{nm} / \mathrm{km}$ dispersion as shown in Fig.4.

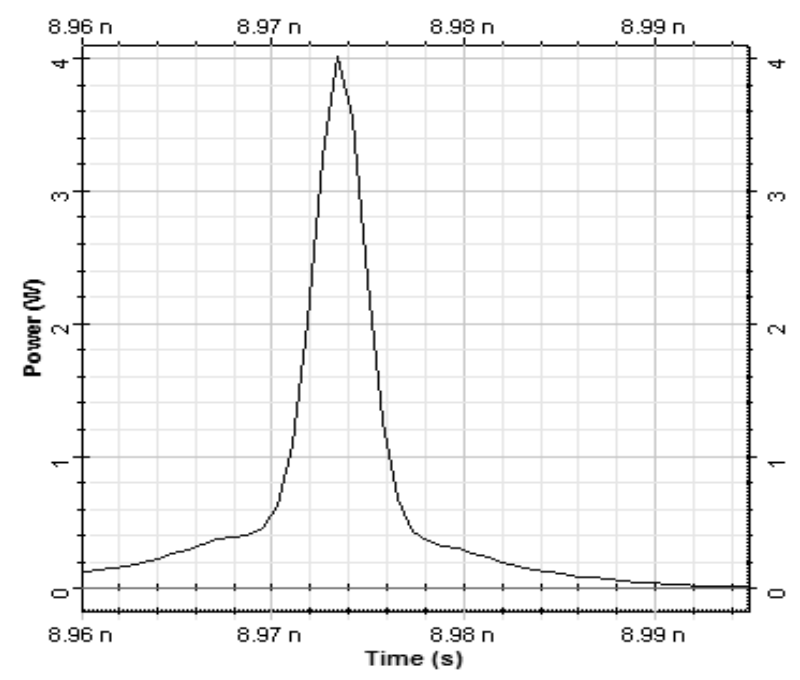

Fig. 4. Pulse shape of pump wave after SMF 
Using the EDFA, the pulse train is boosted by $1 \mathrm{~W}$. Coupler 3 and Coupler 4 with 9\% coupling ratios are fed back into the polarization combiner. In the loop of nonlinear fibers, there are three HNLFs with zero dispersion wavelengths (ZDW): HNLF1 is $1600 \mathrm{~nm}, \mathrm{HNLF} 2$ is $1550 \mathrm{~nm}, \mathrm{HNLF} 3$ is $1500 \mathrm{~nm}$. The length, nonlinear coefficient, and dispersion slope of HNLFs are L $=0.03 \mathrm{~km}, \gamma=26 \mathrm{~W}-1 . \mathrm{km}-1$, slope $=0.03$ $\mathrm{ps} / \mathrm{nm} / \mathrm{km} 2$. The layout was then simulated under different configurations to validate the effectiveness of the proposed scheme. Without using the SMF, results were acquired for 20 spectral lines in $10 \mathrm{dBm}$ power deviation as shown in Fig.5. When the HNLF1 was removed from the scheme, results yielded 24 lines in $10 \mathrm{dBm}$ power deviation as shown in Fig.6. In a third configuration, the whole feedback structure which contains HNLFs was removed to obtain 26 lines in $10 \mathrm{dBm}$ power deviation as shown in Fig.7. In the final configuration which uses the proposed scheme, an evenly spaced 27 lines were acquired in $6 \mathrm{dBm}$ power deviation as shown in Fig.8.

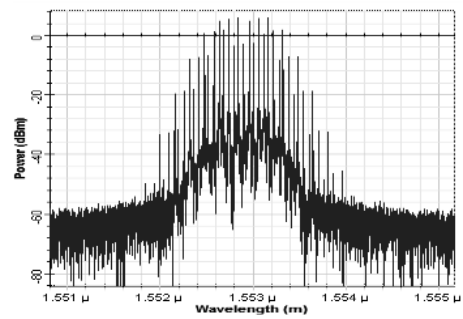

Fig. 5. Response of the system without SMF

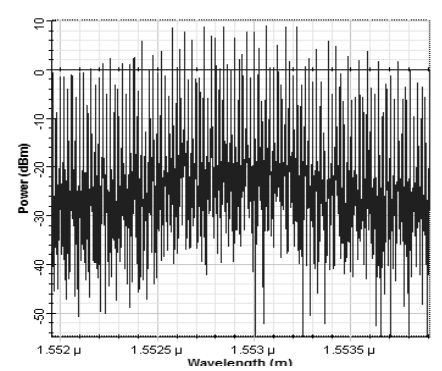

Fig. 7. Response of the system without feedback structure

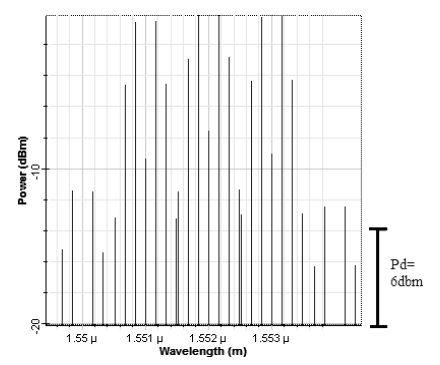

Fig. 8. Response of the proposed scheme

\section{A. Characterization and Optimization of the Comb Responses}

The impact of dispersion on the generation of FWM is evident in the results. As the dispersion of SMF increases, it reduces the number of comb lines as shown in Fig.8. Results show that as the number of pump sources with the same frequency spacing increases, the efficiency of FWM increases linearly. In this paper, the pumps are tuned with $1 \mathrm{~nm}$ and 0.1 $\mathrm{nm}$ frequency spacing to evaluate the performance of optical frequency comb shown in Fig.9 and Fig.10. By using $0.1 \mathrm{~nm}$ the numbers of lines are higher but they are not evenly spaced with each other. This implies that crosstalk can occur during the transmission system. Comparing Fig.9 and Fig.10, it is evident that while using $1 \mathrm{~nm}$ frequency spacing the number of lines increases linearly, but while using $0.1 \mathrm{~nm}$ spacing after five pump sources the spectral lines start reducing in number.

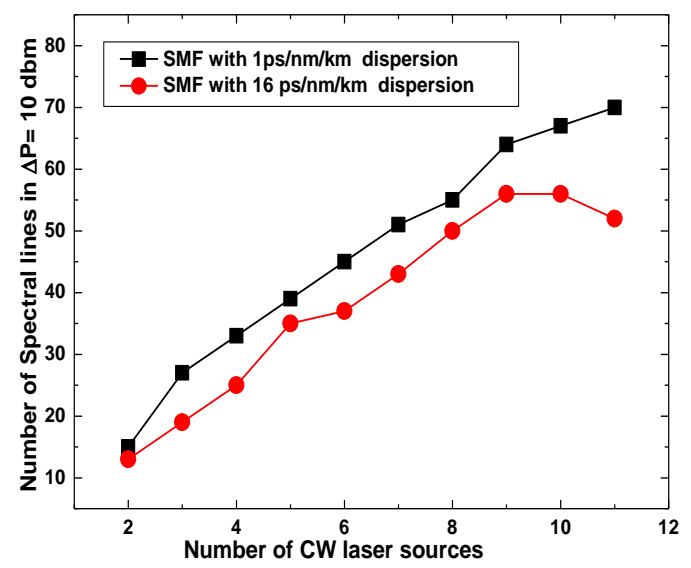

Fig. 9. Number of spectral lines analysis for different pump sources in $1 \mathrm{~nm}$ channel spacing and dispersion effects of SMF

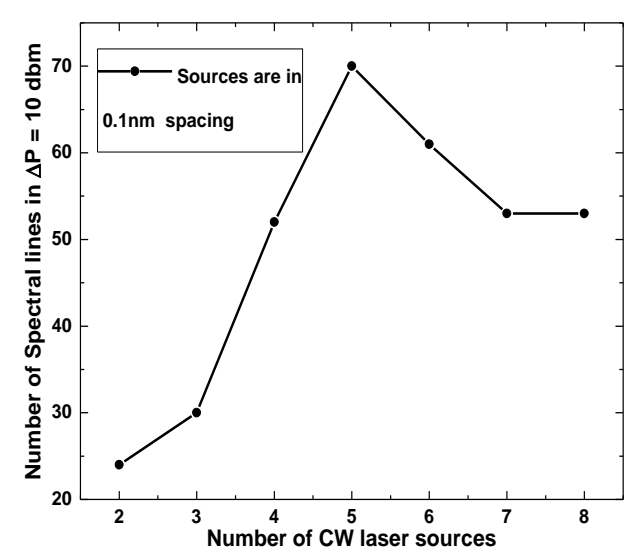

Fig. 10. Number of spectral lines analysis for different pump sources

Next, the impact of pump power on the system was explored. Results show that as the pump power increases, more sidebands are induced in the spectrum due to the effective generation of FWM in highly nonlinear fibers. Fig. 11 clearly shows the effect of varying the pump power from -30 $\mathrm{dBm}$ to $30 \mathrm{dBm}$. Sharp increases in the number of spectral lines were found at pump powers exceeding $20 \mathrm{dBm}$.

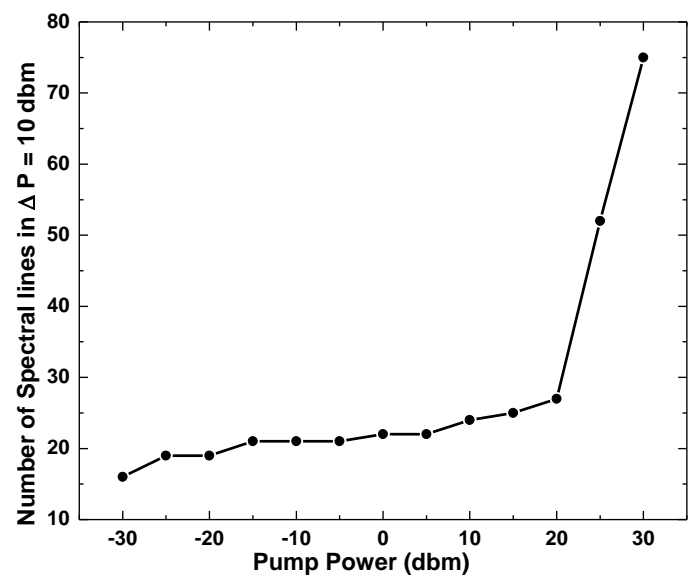

Fig. 11. Correlation between the pump power and spectrum lines

Last but not least, the effect of EDFA length on the generated spectral lines was investigated as shown in Fig.12. The number of frequency lines increases in $10 \mathrm{dBm}$ power deviation as the EDFA length increases. When the EDFA 
length is set to $15 \mathrm{~m}$, a maximum number of 27 spectral lines were achieved for the proposed scheme. Any further increase in the length did not have any major impact. A huge increase in length, however, showed a large drop in the number of spectral line generation. This is largely due to the increased output power for longer lengths of EDFA.

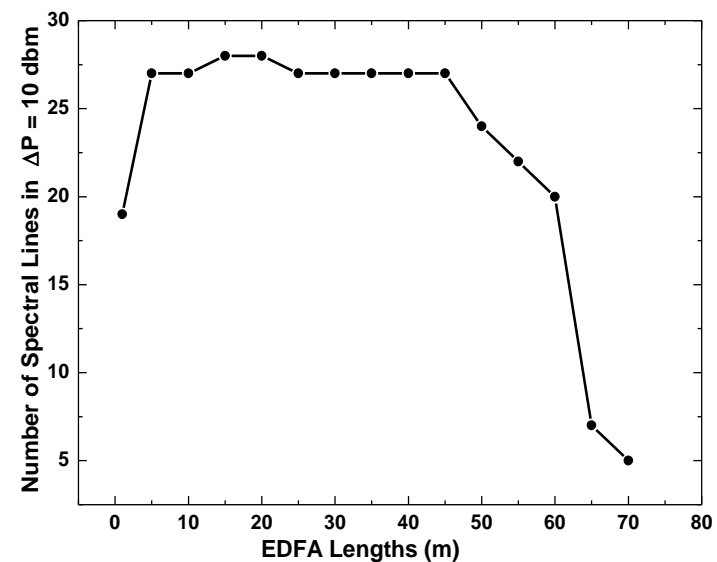

Fig. 12. Variation in number of spectral lines for different EDFA lengths

\section{B. Analysis of Proposed Final Optimized Scheme}

To mitigate the fluctuations of the previous proposed initial scheme, a new scheme is proposed as shown in Fig. 13.

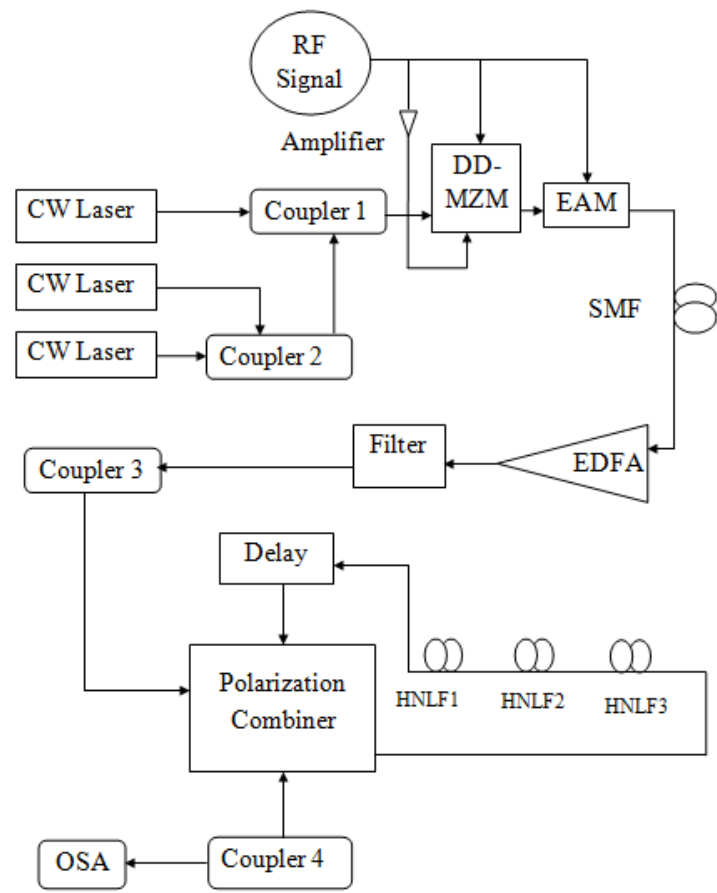

Fig. 13. Proposed Methodology for flat optical comb generation

As shown in Fig.13, three identical pump sources as the previous scheme are now coupled using $3 \mathrm{~dB}$ couplers of 10 $\mathrm{MHz}$ line width and then passed through a cascade of both DD-MZM \& EAM modulators. The DD-MZM component in Optisystem layout is driven by an RF signal of $25 \mathrm{GHz}$ frequency which is split and introduced into the two arms of the modulator. Since the two RF signals have to be of different amplitudes, one of them is first amplified by $-1 \mathrm{~dB}$ gain before it is injected in one arm. The biasing voltages used in these two arms are $-4.65 \mathrm{~V}$ and $-3.1 \mathrm{~V}$. As the DD-MZM operates as both intensity and a phase modulator, there is no need to add any phase shifter. The RF signal is also injected into the EAM modulator which modulated the spectrum with a $1.6 \mathrm{~V}$ peak to peak voltage to modify the sideband signals and flatten the spectrum.

After this spectrum travels through a $1 \mathrm{~km}$ long SMF to suppress Brillouin scattering, it is amplified by the EDFA by 1 $\mathrm{W}$. Here the spectrum picks up ASE, so it is then passed through a filter to get rid of this unwanted noise. Couplers 3 and 4 are using 9\% coupling ratio for feedback into the polarization combiner [16].

The filtered spectrum then finally passes through the feedback loop of $0.03 \mathrm{~km}$ long HNLF with the polarization combiner. The three HNLFs have ZDW at $1600 \mathrm{~nm}, 1550 \mathrm{~nm}$, and $1500 \mathrm{~nm}$ as well as an additional delay inserted. The effective area of all HNLFs is Aeff $=64 \mu \mathrm{m} 2$. Finally, the polarization combiner initiates the FWM phenomenon which is assisting in creating the proposed spectral lines.

\section{Results and Discussion}

Fig.14 shows the results while using only a phase modulator. The system is able to generate about 15 lines but with a high degree of fluctuation in the amplitudes of each line. Such a response would create a lot of problems in a communication system.

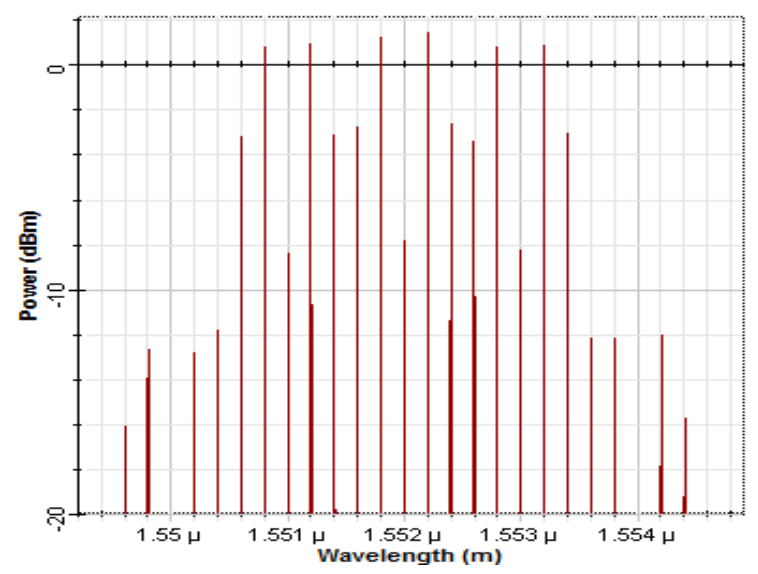

Fig. 14. Comb output using only the phase modulator

Fig.15 illustrates the result using the cascading of single drive MZM and EAM. It is evident here that by cascading the two modulators, the resultant comb has fewer lines but the amplitude deviation is only about $2 \mathrm{dBm}$.

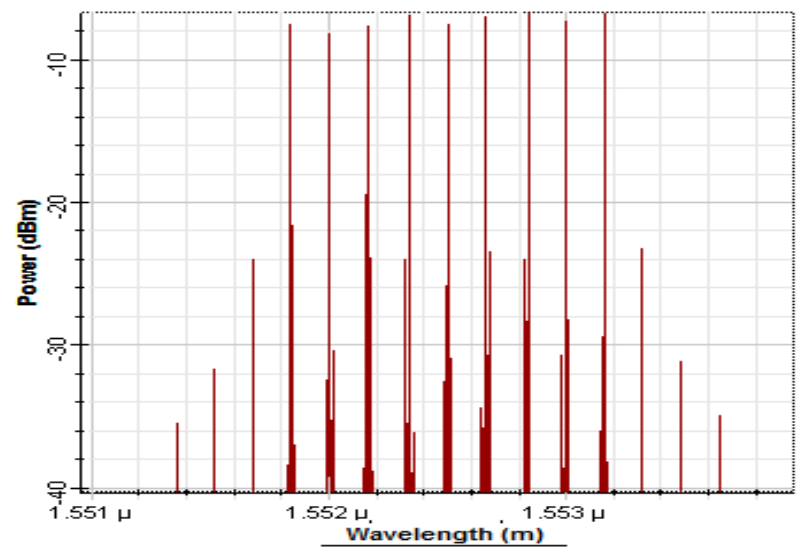

Fig. 15. Response with cascading of single drive MZM and EAM 
Fig.16 is displaying the output for the proposed scheme of cascading the DD-MZM and EAM. Here the number of comb lines is the same as Fig.14 while the amplitude deviation is only $2 \mathrm{dBm}$ which is the same as Fig.15. Clearly, it has the best part of both aforementioned scenarios.

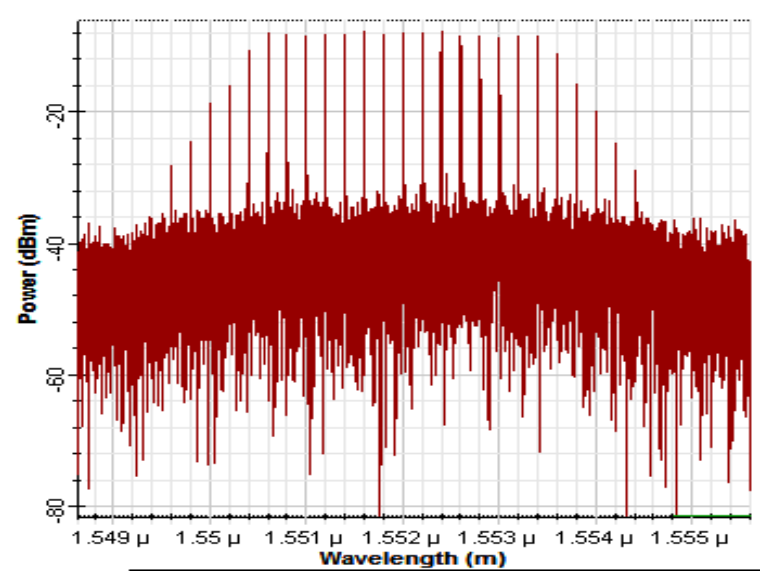

Fig. 16. Proposed scheme response with cascading of dual-drive MZM and EAM

\section{Optimization of Generated Comb Response}

While investigating the system, it is only natural to question the impact of the critical parameters such as the number of pump sources and the amount of channel spacing. When the number of pump sources was varied by keeping the channel spacing at $1 \mathrm{~nm}$, Fig. 17 shows the correlation. It is evident that the system is yielding the best response when 5 pump sources are used. At this point, it is producing 23 flat spectrum lines.

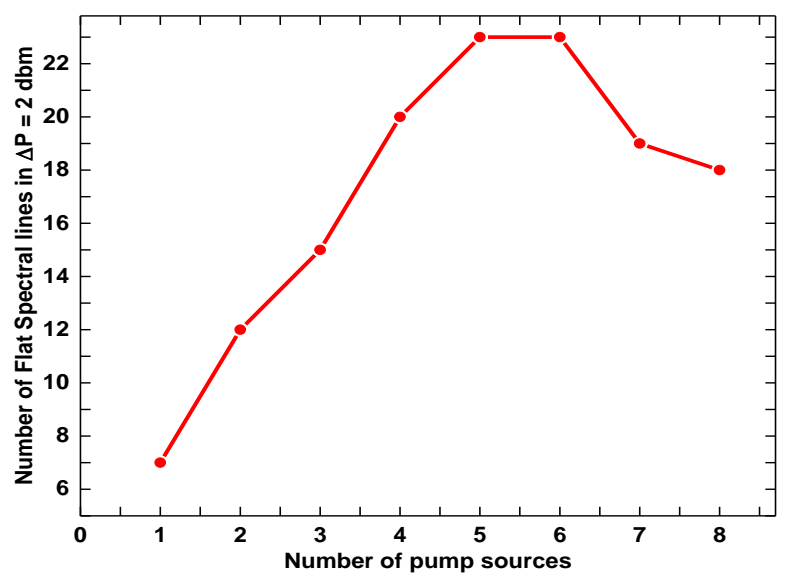

Fig. 17. Correlation of number of pump sources on comb spectrum

As seen in Fig.18, varying the channel spacing between the sources increases the spectral lines as well as having an impact on the flatness of the power deviation. When $1 \mathrm{~nm}$ channel spacing is used, 15 spectrum lines are generated with $2 \mathrm{dBm}$ power deviation.

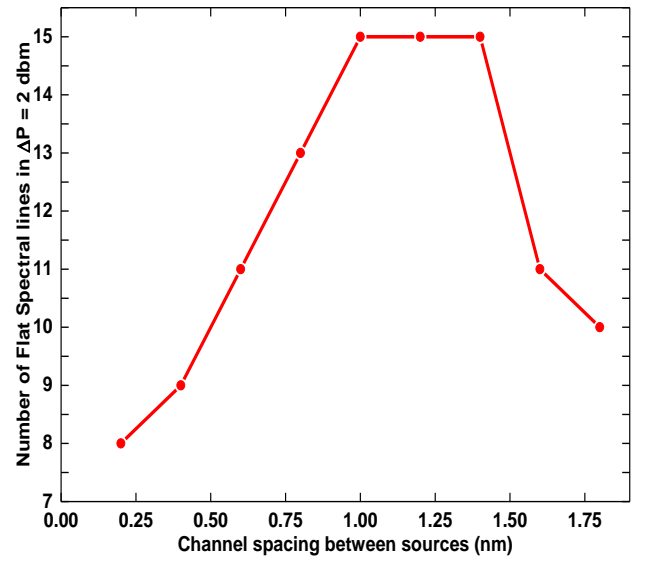

Fig. 18. Correlation of flat spectrum lines and channel spacing

The driving RF signals bandwidth also has an impact on the flatness of the comb spectrum. Fig.8 shows that the maximum of 15 flat spectrum lines can be generated using $25 \mathrm{GHz}$.

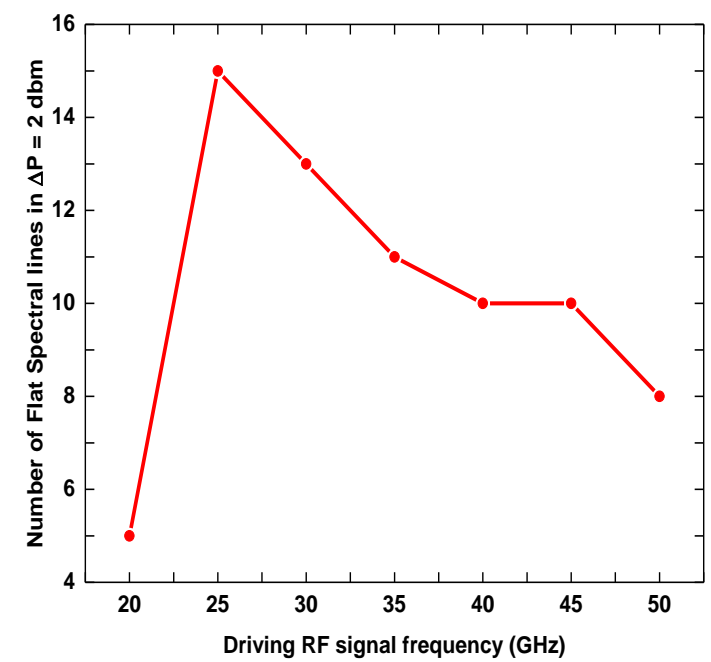

Fig. 19. Correlation between driving RF signals and flat spectral lines

\section{E. Performance Analysis of Comb in Preliminary WDM System}

Previously the comb generator operated for $25 \mathrm{GHz} \mathrm{RF}$ signal to generate maximum and flat comb lines in small power deviation. Now, in the proposed scheme when the 10 $\mathrm{Gb} / \mathrm{s}$ message signal is inserted, the shape of the response changes. Fig.20. shows all the comb lines carrying the same identical message. 


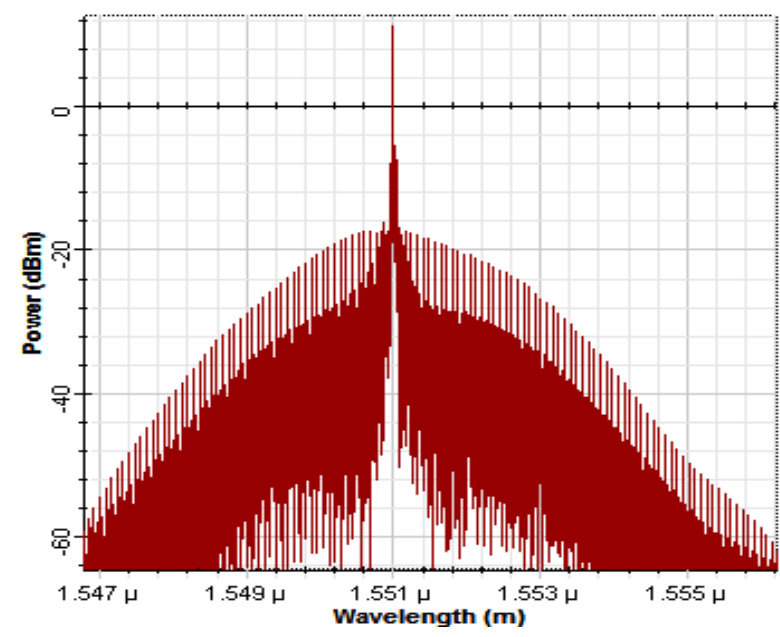

Fig. 20. Response of proposed scheme with $10 \mathrm{~Gb} / \mathrm{s}$ message

The comb lines are then tested as individual channels carrying $10 \mathrm{~Gb} / \mathrm{s}$ signals for evaluating the Eye Diagram, BER and $\mathrm{Q}$ factor. After a transmission of $1 \mathrm{~km}$ distance, the results are shown in Fig. 21 for the first 5 modes. It is evident in Fig.21 that even though all tested channels have acceptable Eye Diagrams, the performance of each individual channel is somewhat different due to the nonlinear behavior of the FWM.
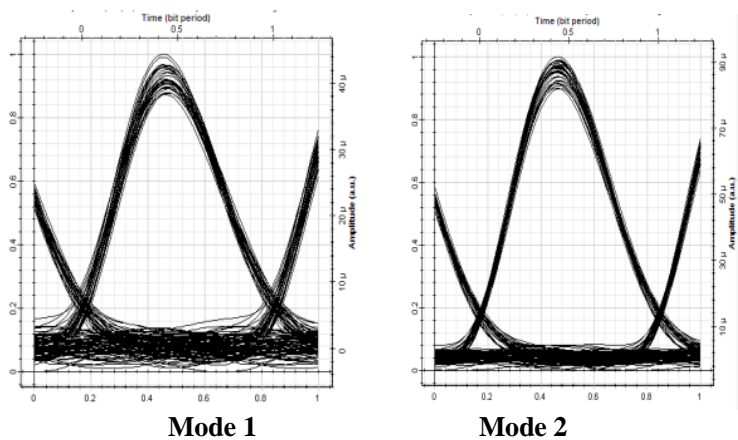

Mode 2
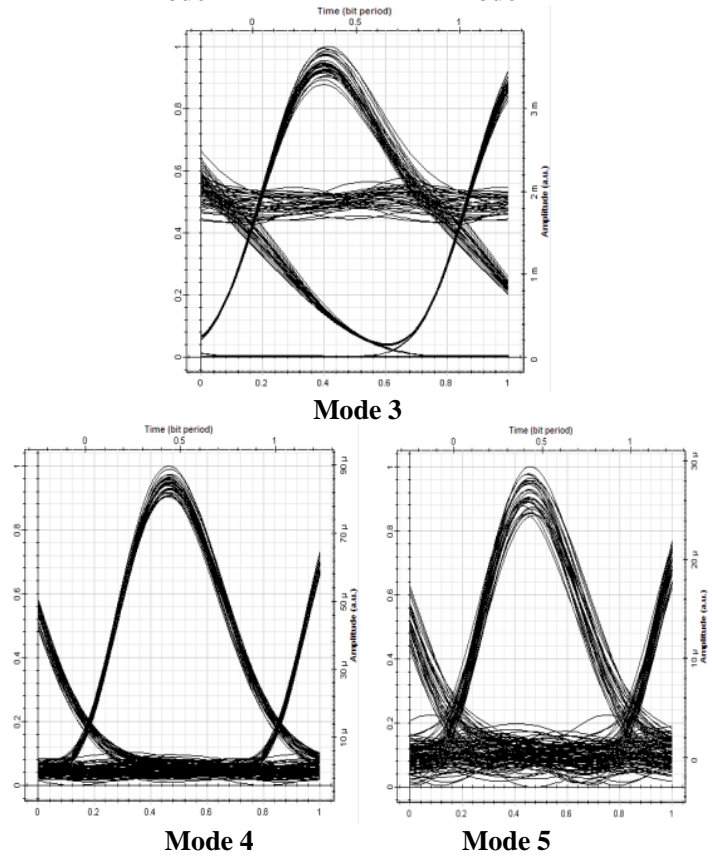

Fig. 21. Eye diagrams of 5 modes for $1 \mathrm{~km}$ transmission

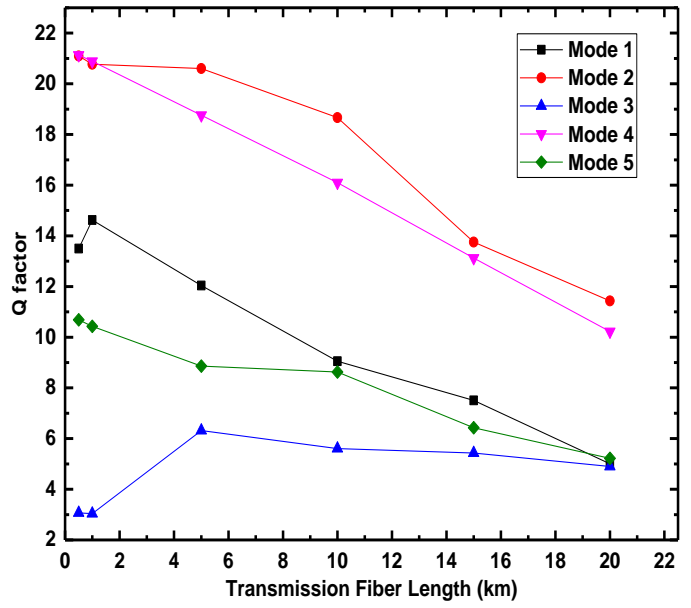

Fig. 22. Variation of Q factor according to transmission length

Although the $\mathrm{Q}$ factor is inversely proportional to the length of the transmission line, the nonlinear behavior of the FWM is showing an interesting response for the first 5 channels in Fig. 22. When the responses of the first 5 modes are compared with an increase in transmission length, the best combined result is evident at $1 \mathrm{~km}$ fiber length. This proves that the effect of FWM in each mode is different and thus the performance needs to be optimized based on the number of channels.

A third performance parameter BER was also compared for the 5 modes and the results showed a similar behavior in Fig. 23 where the combined best results were found at $1 \mathrm{~km}$ transmission.

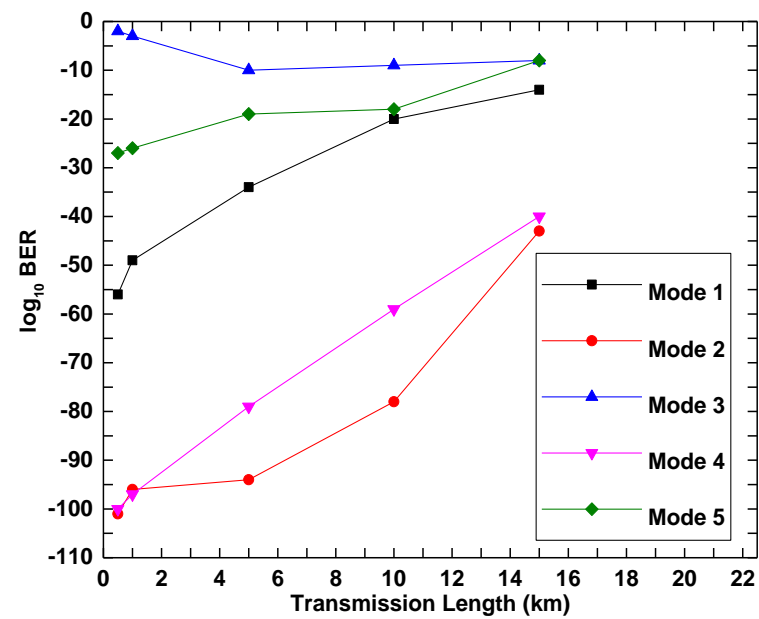

Fig. 23. Variation of BER according to transmission length

\section{CONCLUSION}

This paper has presented the results of a simulation scheme using cascaded DD-MZM, EAM, and nonlinear optical fiber loops to produce an evenly spaced flat optical comb spectrum applicable for a WDM fiber optic communication system. The initial goal was to yield a high number of channels to increase the capacity of the WDM, but the uneven power deviation in the results due to the nonlinear aspects of the design motivated the researchers to seek further optimization which was successful in reducing the power deviation down to $2 \mathrm{dBm}$, but at the expense of number of channels/modes produced in the comb. The final results were quite promising as the comb was evaluated for a WDM implementation where it was 
successful in transmitting $10 \mathrm{~Gb} / \mathrm{s}$ date in each of the five channels up to a distance of $1 \mathrm{~km}$. The results of this paper in terms of spectral lines can be compared with the recent research works to mention the uniqueness of the proposed responses.

TABLE I. COMPARISON WITH RECENT RESEARCH WORK

\begin{tabular}{|c|c|}
\hline Proposed Paper Results & Recent Published Work Results \\
\hline $\begin{array}{l}\text { 1. OFC generation using a cascade } \\
\text { of DD-MZM \& EAM in a nonlinear } \\
\text { feedback structure determined } 15 \\
\text { flat comb lines among } 25 \text { lines in } 2 \\
\text { dBm power deviation. }\end{array}$ & $\begin{array}{l}\text { 1. Using a single drive dual parallel } \\
\text { MZM with two different amplitudes, } \\
\text { RF signals have been used to } \\
\text { generate 5-7 flat lines in } 18 \mathrm{dBm} \\
\text { [17]. } \\
\text { 2. Cascading of two intensity } \\
\text { modulators has generated } 9 \text { flat } \\
\text { spectral lines in } 2 \mathrm{dBm} \text { power } \\
\text { deviation [18]. } \\
\text { 3. By incorporating the } \\
\text { optoelectronic oscillation loop with } \\
\text { cascaded modulators in optical comb } \\
\text { generator has produced } 11 \text { ultra flat } \\
\text { lines [19]. } \\
\text { 4. Cascading of EAM and two PM } \\
\text { obtained 11flat lines in } 2 \mathrm{dBm} \text { power } \\
\text { deviation [20]. }\end{array}$ \\
\hline $\begin{array}{l}\text { 2. The OFC generation using FWM } \\
\text { in multiple nonlinear fibers loop } \\
\text { obtained } 27 \text { lines in } 6 \mathrm{dBm} \text { power } \\
\text { deviation. }\end{array}$ & $\begin{array}{l}\text { 1. OFC generation using multiple } \\
\text { FWM by making use of EDFA in } \\
\text { nonlinear fiber experimentally } \\
\text { obtained } 23 \text { lines in } 10 \mathrm{dBm} \text { power } \\
\text { deviation [21]. }\end{array}$ \\
\hline
\end{tabular}

\section{References}

[1] P. Del'Haye, A. Schliesser, O. Arcizet, T. Wilken, R. Holzwarth and T. J. Kippenberg, "Optical frequency comb generation from a monolithic microresonator," Nature 450, 2007, pp.1214-1217

[2] R. Ullah, B. Liu, Q. Zhang, Q. Tian, F. Tian, Z. Qu, C. Yan, M. Khan, I. Ahmad and X. Xin, "Optical frequency comb generation with high toneto-noise ratio for large-capacity wavelength division multiplexed passive optical network", Optical Engineering, vol. 54, no. 11, 2015, pp. 11610.

[3] Yang, Ting et al. "Comparison Analysis Of Optical Frequency Comb Generation With Nonlinear Effects In Highly Nonlinear Fibers". Opt. Express 21.7, 2013

[4] Zhou, Rui et al. "Injection Locked Wavelength De-Multiplexer for Optical Comb-Based Nyquist WDM System". IEEE Photonics Technology Letters, vol.27, no.24, 2015, pp. 2595-2598.

[5] Jianrui Li, Jianrui Li et al. "Scheme for Generation of flat top and high signal-to-noise ratio optical frequency comb". Chinese Optics Letters 13.1, 2015

[6] Nagarjuna, K.P, Shankar Kumar Selvarajaa, and V.R Supradeepa, "Generation Of Tunable, High Repetition Rate Frequency Combs With Equalized Spectra Using Carrier Injection Based Silicon Modulators", Proc. of SPIE, vol. 9752, 2016.

[7] Rifat Nazneen, Ebad Zahir, "Analysis and Optimization of an Optical Frequency Comb for Multiple Channel Transmission in a Fiberoptic System" 3rd International Conference on Electrical Engineering and Information Communication Technology (iCEEiCT 2016) at MIST, Mirpur Cantonment, Dhaka

[8] R.Nazneen, E. Zahir, "Cascading of Dual Drive Mach Zehnder Modulator and Electro Absorption Modulator for Producing an Optical Comb", 9th International Conference on Electrical and Computer Engineering (ICECE 2016) at BUET, Dhaka
[9] O. Aso, M. Tadakuma and S. Namiki, "Four-Wave Mixing in Optical Fibers and Its Applications", Furukawa Review, no. 19, 2000.

[10] A. Cerqueira Sodre, J. Chavez Boggio, A. Rieznik, H. HernandezFigueroa, H. Fragnito and J. Knight, "Highly efficient generation of broadband cascaded four-wave mixing products", Opt. Express, vol. 16, no. 4, 2008, pp. 2816

[11] Morant, Maria et al. "Dual-Drive Linbo_3 Interferometric MachZehnder Architecture with Extended Linear Regime For High Peak-ToAverage OFDM-Based Communication Systems", Opt.Express 19.26, 2011

[12] Kumar, Shiva and M. Jamal Deen, "Fiber Optic Communications", Hoboken: Wiley, 2014.

[13] F. Zhang, J. Wu, Y. Li and J. Lin, "Flat optical frequency comb generation and its application for optical waveform generation", Optics Communications, vol. 290, pp. 37-42, 2013.

[14] Dong, Qimeng et al. "Flat Frequency Comb Generation Based On Efficiently Multiple Four-Wave Mixing Without Polarization Control". Photonic Sens 6.1, 2015, pp.85-89.

[15] Li, Jiabao et al. "Enhancement of Cascaded Four-Wave Mixing Via Optical Feedback". Opt. Express 20.20, 2012

[16] A. Antikainen and G. Agrawal, "Dual-pump frequency comb generation in normally dispersive optical fibers", Journal of the Optical Society of America B, vol. 32, no. 8, 2015, pp. 1705

[17] Shang, Lei et al. "A Flexible And Ultra-Flat Optical Frequency Comb Generator Using A Parallel Mach-Zehnder Modulator With A Single DC Bias". Optics Communications 356, 2015, pp. 70-73

[18] X. Zhou, X. Zheng, H. Wen, H. Zhang, Y. Guo, B. Zhou, "All optical arbitrary waveform generation by optical frequency comb based on cascading intensity modulation", Opt. Commun. 284, 2011, pp. 3706 3710

[19] Dai, Jian et al. "Self-Oscillating Optical Frequency Comb Generator Based on an Optoelectronic Oscillator Employing Cascaded Modulators". Opt. Express 23.23, 2015: 30014

[20] Fangzheng Zhang n, Jian Wu, Yan Li, Jintong Lin, "Flat optical frequency comb generation and its application for optical waveform generation", Optics Communications 290, 2013, pp. 37-42.

[21] Suzanne A.S.Melo, et.al, "Optical frequency comb based on Multiple Four- Wave Mixing and Erbium doped fiber", REVISTA TELECOMUNICACOES, vol. 15, No. 2, October 2013

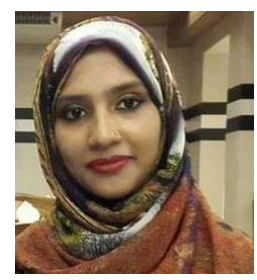

Rifat Nazneen was born in 13th September, 1992 in Dhaka , Bangladesh. She completed both her M.Sc and B.Sc in Electrical and Electronic Engineering from American International University- Bangladesh (AIUB). She has research interests on Optical Communicatons, Optoelectronics, Image Processing etc.

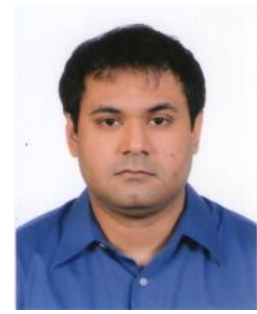

Ebad Zahir is currently employed at American International University- Bangladesh (AIUB) as a Senior Assistant Professor for the Faculty of Engineering and Special Assistant for the Department of Electrical and Electronic Engineering. 